\title{
Research on Pump Volute Design Method Using CFD
}

\author{
Sunsheng Yang, Fanyu Kong, and Bin Chen \\ Research Center of Fluid Machinery Engineering and Technology, Jiangsu University, Zhenjiang 212013, China \\ Correspondence should be addressed to Sunsheng Yang, yangsunsheng@126.com
}

Received 27 September 2010; Accepted 31 March 2011

Academic Editor: David Japikse

Copyright (C) 2011 Sunsheng Yang et al. This is an open access article distributed under the Creative Commons Attribution License, which permits unrestricted use, distribution, and reproduction in any medium, provided the original work is properly cited.

\begin{abstract}
Volute is an important hydraulic part of centrifugal pump, hydraulic loss within pump volute takes up a large part of total hydraulic loss within pump, thus appropriate design of pump volute has significant meaning to centrifugal pump performance. In this paper, numerical method was adopted to investigate volute main geometric parameters, including volute throat area, volute crosssection shape, design rule of spiral development area, and radial gap between impeller and volute tongue to pump performance. A design method of high-efficiency pump volute is developed through the influence of volute main geometric parameters to pump performance. This paper could provide theoretical guidance to high-efficiency pump volute design.
\end{abstract}

\section{Introduction}

Pumps are energy-consuming devices people used in daily life, the efficiency improvement of pumps have significant meaning to energy conservation. Volute as a stationary hydraulic part of pump plays an important role to the pump performance; appropriate design of pump volute plays an important role in pump efficiency improvement.

Zhao and $\mathrm{Xu}$ [1] overviewed current research on volute and provided several suggestions on further research. Deng and Chu [2] investigated volute throat area to the centrifugal pump performance and drew a conclusion that the decrease of volute throat area could narrow down pump high efficiency range, and steepen its $Q-H$ curve. Chen and Wei [3] investigated the design rule of hydraulic reaction turbine volute spiral development areas and proposed a high-efficiency volute spiral development areas design rule. Research on geometric parameters to the performance of pump mainly focuses on one geometric parameter; besides, some of the geometric parameters are very hard to pursue experimental research. Centrifugal design software and CFD technology provide an effective tool to investigate geometric parameters to pump performance.

In this study, a single-stage centrifugal pump was desi-gned. Effects of volute main geometric parameters like throat area, cross-section shape, design rule of spiral development areas, and radial gap between impeller and volute tongue to the performance of pump were carried out using numerical method. The curves of pump with different geometric parameters were acquired. Through analysis of these parameters performance curves, a high-efficiency volute design method was developed.

\section{Pump Geometry}

The focus of the investigation is a single-stage centrifugal pump. Its design parameters are as follows: $Q=210 \mathrm{~m}^{3} / \mathrm{h}, H$ $=100 \mathrm{~m}$, and $n=3000 \mathrm{r} / \mathrm{min}$. Table 1 lists the main geometric parameters of the designed pump. Figure 1 provides a general view of the designed pump.

\section{Numerical Investigation}

ANSYS-CFX is a commercial 3D Navier-stokes CFD code that utilises a finite-element-based finite-volume method to discretise the transport equations. It is a fully implicit solver, thus it creates no time step limitation and is considered easy to implement. It is also a coupled solver meaning that the momentum and continuity equations are solved simultaneously. This approach reduces the number of iterations required to obtain convergence and no pressure correction term is required to retain mass conversion, leading to a more robust and accurate solver. 


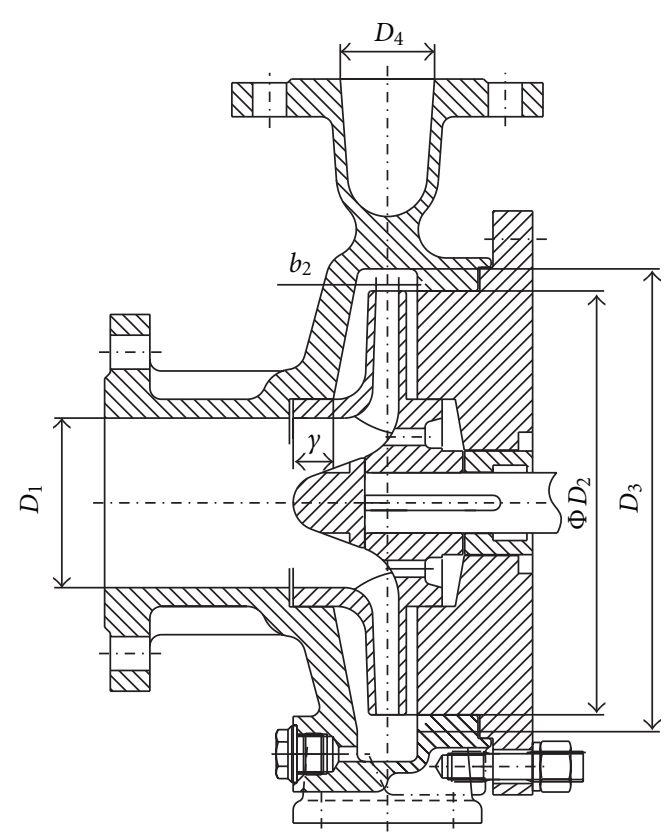

Figure 1: General view of the designed pump.

TABle 1: Main geometric parameters of the designed pump.

\begin{tabular}{ccccccccc}
\hline$D_{1}$ & $z$ & $\Phi$ & $\beta_{2}$ & $D_{2}$ & $b_{2}$ & $\gamma$ & $D_{3}$ & $D_{4}$ \\
\hline 100 & 7 & 120 & 25 & 280 & 16.63 & 23 & 308 & 80 \\
\hline
\end{tabular}

The fluid within pump front and back chambers is usually neglected during CFD simulation $[4,5]$. In order to get a more accurate result, all fluid within the pump control volume was modeled as indicated in Figure 3(c) in this paper. So the calculated efficiency included hydraulic efficiency and volumetric efficiency. The mechanical efficiency caused by the friction of mechanical seal and bearings were neglected in the calculation of efficiency. The fluid was split into five component parts, they were pump inlet pipe, impeller passage, volute, and front and back chambers. This separation allows each mesh to be generated individually and tailored to the flow requirements in that particular component. To get a relatively stable inlet and outlet flow, four-times of the pipe diameter has been extended in the pump inlet and outlet section.

3.1. Mesh Generation. ICEM-CFD was used to generate structured hexahedral grid for each component part. Denton and Daws [6] have observed that 300,000 nodes are required for adequate representation of viscous effects with shock waves and tip leakage, but also noted that useful comparisons can be made with 100,000 nodes. Miner's published work [7] and Spence and Amaral-Teixeira [8] indicate that relatively coarse impeller models (20,000-40,000 nodes) can be used to predict performance with some accuracy. Denton has also observed that the 22,000 grid per blade passage is sufficient for a calculation with approximate allowance for viscous effects. A grid independent study of this pump was performed, Figure 2 shows the variation of efficiency with

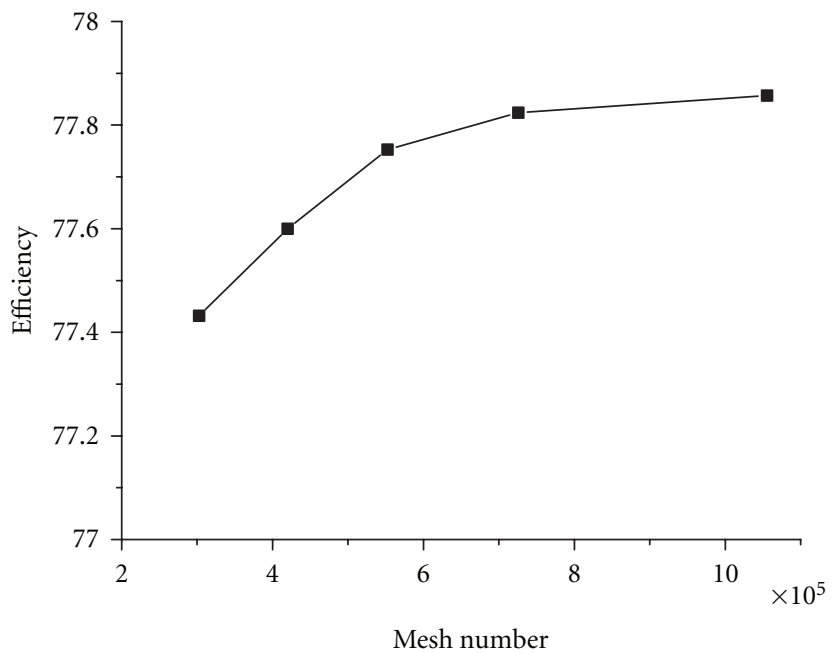

FIgURE 2: Efficiency versus mesh number curve.

mesh number, as could be seen that the variation of efficiency was within $0.5 \%$ as mesh numbers ranged from 500,000 to 100,000 . The final mesh number of inlet pipe, impeller, front chamber, back chamber, and volute is $98312,295240,84$ 835, 61628 , and 375626 , respectively, and the total number of mesh is 915641. For other model's simulation, only the volute was changed and the mesh numbers were almost the same.

Care is taken in the pump inlet mesh generation in order that the mesh between the interface of inlet pipe and front chamber leakage is almost the same. The mesh near the boundary wall is refined to catch the boundary layer effects. Due to the complexity of generating a structured mesh based on geometry, great efforts have been taken in the mesh generation of volute. Figure 3 gives a general view of the generated meshes.

3.2. Solution Parameters. The turbulence was simulated with RNG k- $\varepsilon$ turbulence model. The advection scheme was set to high resolution. The convergence criterion was $10^{-5}$. All the wall surface roughness within the control volume were set to $50 \mu \mathrm{m}$. The inlet and outlet boundary condition were set to static pressure inlet and mass flow rate outlet [8]. By changing mass flow rate, the performance curves of pump were acquired.

As the motion of the impeller blades relative to the stationary volute was central to the investigation, the analysis must involve multiple frames of reference. The suction inlet and volute were set in stationary frame and the impeller was set in rotary frame. The interfaces between two stationary components, rotary and stationary components, were set to general grid interface and rotor stator interface, respectively.

\section{Volute Throat Area}

In the research of volute throat area to pump performance, four different volute throat areas ranging from $3085 \mathrm{~mm}^{2}$ to $4629 \mathrm{~mm}^{2}$ are investigated, the investigated cross-section 


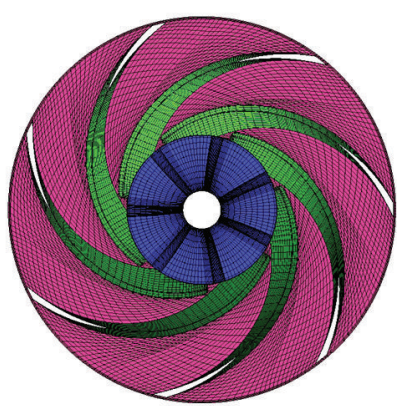

(a)

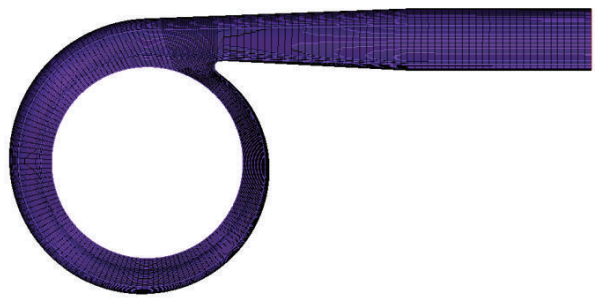

(b)

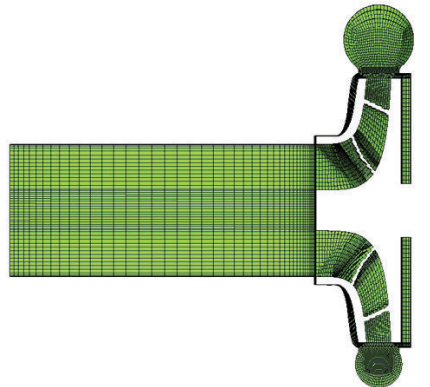

(c)

Figure 3: (a) Meshes within impeller. (b) Meshes within volute. (c) Meshes of a cross-section.

TABLE 2: List of pump BEPs at different volute throat areas.

\begin{tabular}{lcccc}
\hline Volute throat area & Capacity $\left(\mathrm{m}^{3} / \mathrm{h}\right)$ & Efficiency $(\%)$ & Head $(\mathrm{m})$ & Shaft power $(\mathrm{kW})$ \\
\hline $3085 \mathrm{~mm}^{2}$ & 199.2 & 77.83 & 99.80 & 69.53 \\
$3472 \mathrm{~mm}^{2}$ & 215.8 & 77.41 & 95.20 & 72.25 \\
$3968 \mathrm{~mm}^{2}$ & 215.8 & 77.39 & 97.80 & 71.96 \\
$4629 \mathrm{~mm}^{2}$ & 232.4 & 76.47 & 89.69 & 74.21 \\
\hline
\end{tabular}

TABLE 3: List of pump BEPs at different volute cross-section shapes.

\begin{tabular}{lcccc}
\hline Cross-section shape & Capacity $\left(\mathrm{m}^{3} / \mathrm{h}\right)$ & Efficiency $(\%)$ & Head $(\mathrm{m})$ & Shaft power $(\mathrm{kW})$ \\
\hline Round & 215.8 & 77.44 & 94.90 & 71.99 \\
Horseshoe shaped & 215.8 & 76.39 & 93.57 & 71.96 \\
Trapezoid & 215.8 & 75.38 & 92.35 & 71.97 \\
Rectangular & 199.2 & 75.47 & 96.12 & 69.07 \\
\hline
\end{tabular}

TABLE 4: List of pump BEPs with different volute design rules.

\begin{tabular}{lcccc}
\hline Design rule & Capacity $\left(\mathrm{m}^{3} / \mathrm{h}\right)$ & Efficiency $(\%)$ & Head $(\mathrm{m})$ & Shaft power $(\mathrm{kW})$ \\
\hline Constant swirl & 215.8 & 76.92 & 94.08 & 71.85 \\
Constant velocity & 232.4 & 76.36 & 89.59 & 74.23 \\
Radius linear & 232.4 & 75.92 & 88.78 & 73.98 \\
Spline1 & 232.4 & 76.25 & 89.29 & 74.08 \\
Spline2 & 332.4 & 73.96 & 86.94 & 74.36 \\
\hline
\end{tabular}

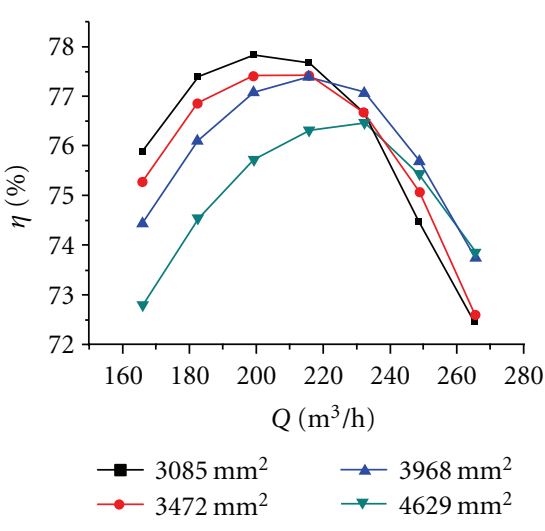

(a)

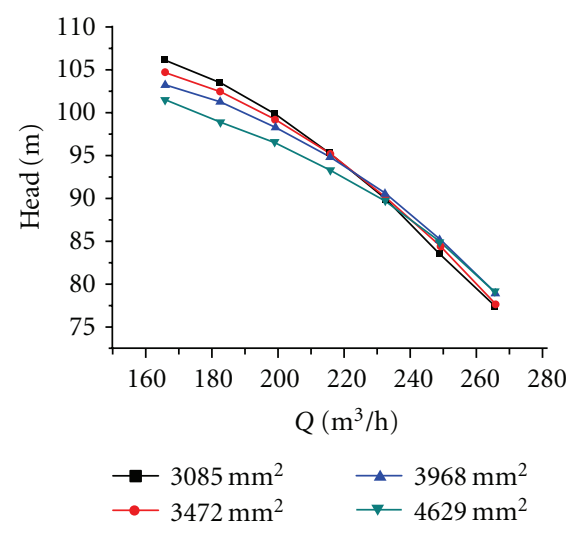

(b)

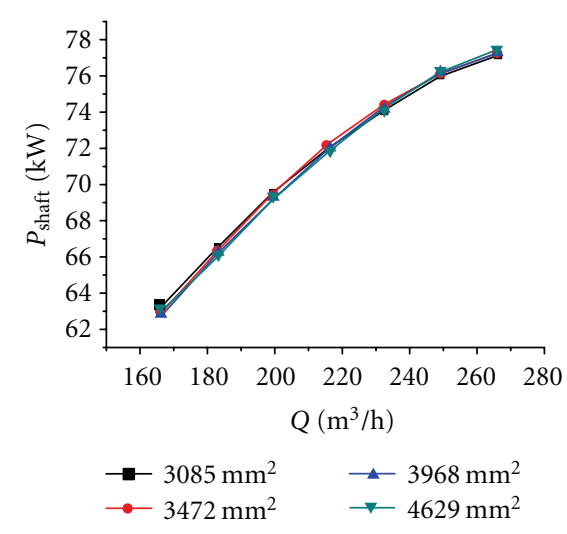

(c)

Figure 4: (a) Q- $\eta$ curve, (b) $Q-H$ curve, and (c) $Q-P_{\text {shaft }}$ curve. 


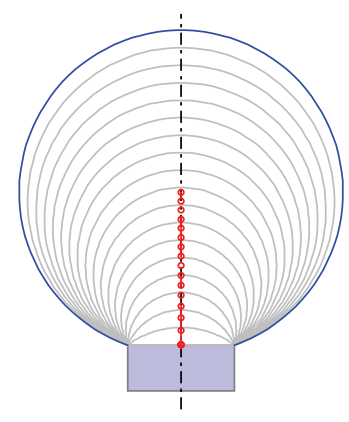

(a) Round

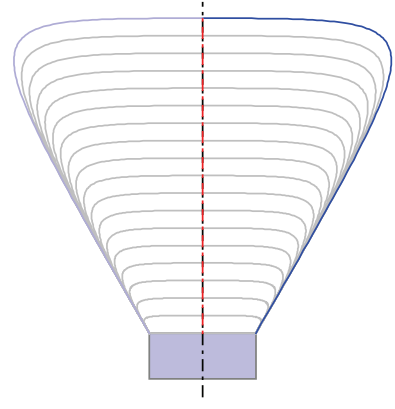

(b) Horseshoe shaped

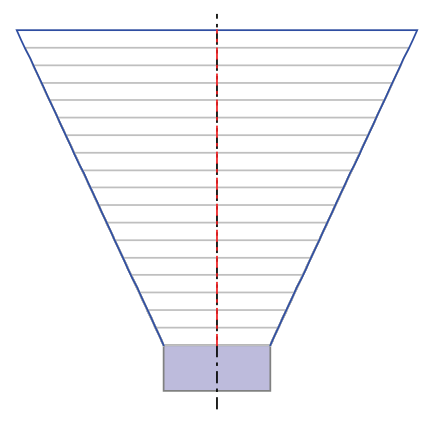

(c) Trapezoid

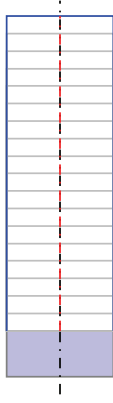

(d) Rectangular

Figure 5: Four type of cross-section shapes.

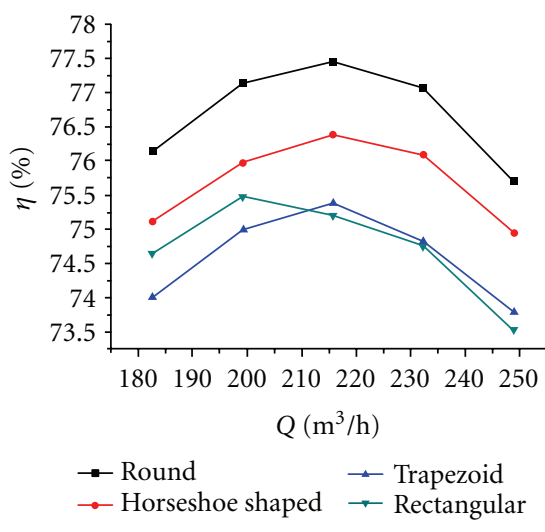

(a)

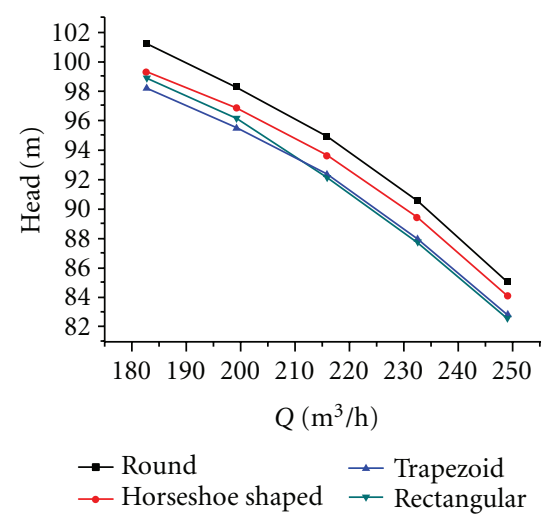

(b)

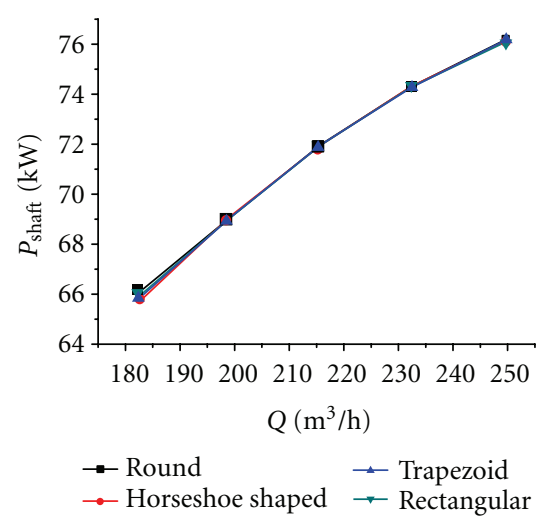

(c)

FIgURe 6: (a) Q- $\eta$ curve, (b) $Q-H$ curve, and (c) $Q-P_{\text {shaft }}$ curve.

shape is round, the spiral development areas is designed according to constant circumferential velocity rule, and all other parameters remain unchanged for the purpose of comparison.

Performance curves of the pump with different volute throat areas were acquired and plotted in Figure 4. Table 2 lists the best efficiency points' (BEP) efficiency, head, and shaft power of different schemes.

As shown in Figure 4(a) and Table 2, with the increase of volute throat area pump BEP moves to large capacity and slightly decreases. Figure 4(b) shows pump pressure head versus flow curve becomes increasingly flat with the increase of volute throat area. Figure 4(c) indicates that pump required shaft power varies at a neglectable amount with different volute throat area.

\section{Volute Cross-Section Shape}

Volute cross-section shape is an important geometric parameter in volute design. In the research of volute cross-section shape to pump performance, the volute throat area remains constant at $3968 \mathrm{~mm}^{2}$, the design of each cross-section areas obeys the constant circumferential velocity rule, and all other parameters like volute outlet pipe diameter and volute base circle diameter are kept constant, only the cross-section shape is changed. Figure 5 indicates the investigated four types of volute cross-section shape usually used in pump design.

Performance curves of the pump with different volute cross-section shapes were acquired and plotted in Figure 6. Table 3 lists the BEP's efficiency, head and shaft power of different schemes.

As is indicated in Figure 6(a) and Table 3, the highest efficiency is reached when volute cross-section shape is round, and the second highest cross-section shape is horseshoe shaped which is $1.05 \%$ lower than that of round-shaped cross-section. Pumps with rectangular- and trapezoid-shaped volute have almost the same efficiency. This indicates that round cross-section shape is the best option for the pump cross-section design from the efficiency point of view.

Figure 6(b) shows that the round-shaped volute crosssection pump generates the highest pressure head. The second highest pressure head is pump with horseshoeshaped cross-section. The pressure head of rectangularand trapezoid-shaped cross-section pump is almost the same. Figure 6(c) indicates that the pump shaft power with different volute cross-section shapes varies little which is neglectable. Detailed hydraulic loss distribution shows that it is the decrease of hydraulic loss within round-shaped volute 


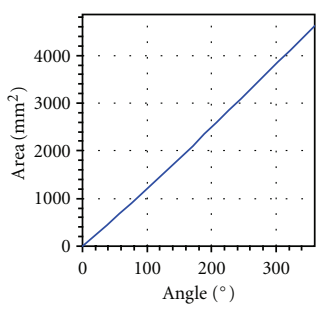

(a) Constant swirl

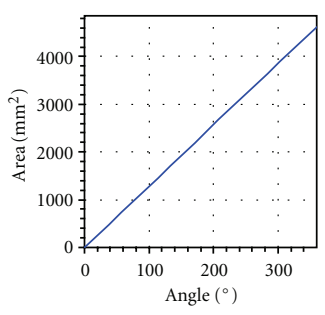

(b) Constant velocity

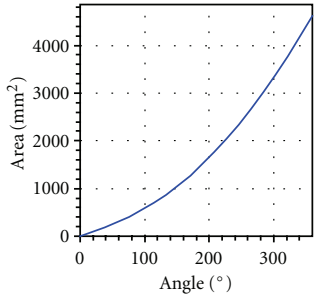

(c) Radius linear

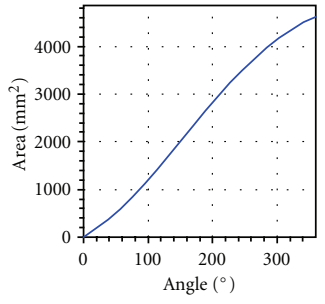

(d) Splinel

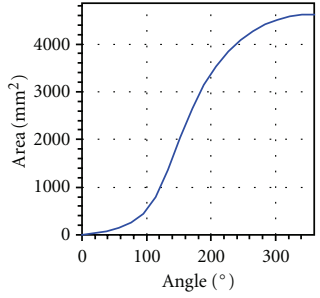

(e) Spline2

Figure 7: Different design rules of spiral development areas.

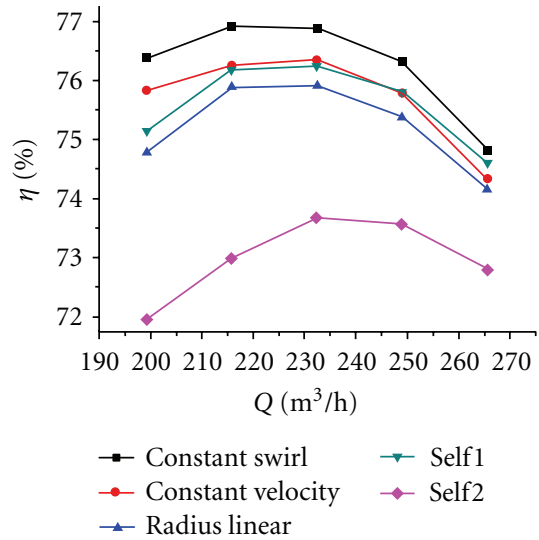

(a)

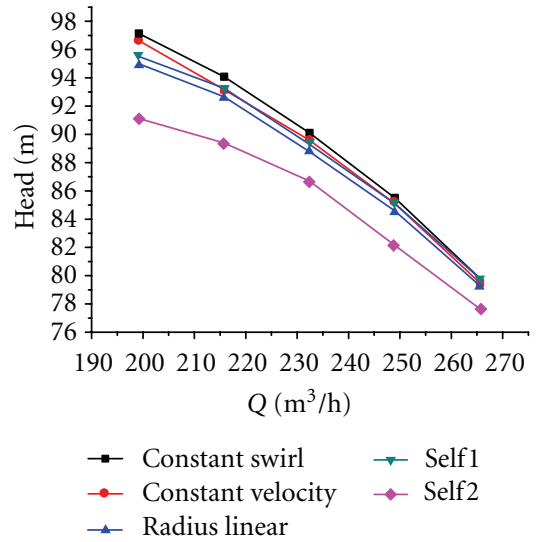

(b)

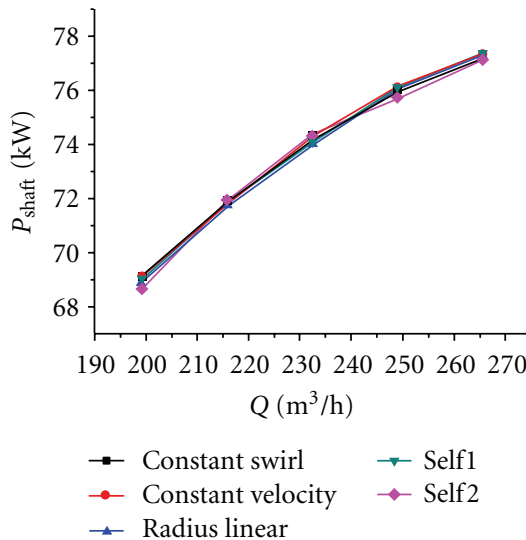

(c)

Figure 8: (a) $Q-\eta$ curve, (b) $Q-H$ curve, and (c) $Q-P_{\text {shaft }}$ curve.

that causes the increase of its pressure head and efficiency when compared with other shaped cross-section.

\section{Design Rule of Spiral Development Areas}

It is widely accepted that the design of volute spiral development areas should obey constant swirl and constant circumferential velocity in pump volute design $[9,10]$. Recently, other types of rules have been reported to generate higher efficiency [3]. In this research, five types of volute spiral development areas which are constant swirl, constant velocity, radius linear (radius increases linearly from volute cutwater to volute throat area), and two self-designed splines as indicated in Figure 7 are investigated. The volute throat area remains constant at $4628 \mathrm{~mm}^{2}$, the investigated crosssection shape is round, and all other parameters like volute outlet pipe diameter and volute base circle diameter are kept constant.

The performance curves of pump with different volute design rules were acquired and plotted in Figure 8. Table 4 lists the best efficiency points' (BEP) efficiency, head, and shaft power of different schemes.

As is demonstrated in Figure 8(a) and Table 4, pump highest efficiency is reached when the volute spiral development areas obeys the constant swirl rule, which is $0.53 \%$ higher that of the constant velocity rule. The efficiency of pump with volute designed according to constant velocity and splinel is almost the same. The one that has the lowest efficiency is spline 2 which has large cross-section area in the volute outlet areas of spiral part.

Figures $8(\mathrm{~b})$ and $8(\mathrm{c})$ shows the required shaft power of the five design rules is almost the same. Among the five investigated design rules, pump with volute designed by spline2 rule has the lowest pressure head.

\section{Radial Gap between Impeller and Volute Tongue}

The radial gap $\left(\Delta \mathrm{r}=\left(D_{3}-D_{2}\right) / 2\right)$ between impeller and volute tongue is assumed to be a sensitive parameter in pump design. Kurokawa et al. [11] report that this radial gap when too small could increase pump efficiency, deteriorate pump vibration characteristics, and cause cavitation to happen near volute tongue. As the radial gap is increased, pump vibration and cavitation characteristics will be improved; however, its efficiency will decrease. Guan [9] indicates that there is an optimal radial gap to the pump volute design. In the research of this radial gap to pump performance, the volute throat area is $4628 \mathrm{~mm}^{2}$, the volute cross-section shape is round, and the design of spiral development areas obeys the constant swirl rule, only the radial gap is changed.

The performance curves of the pump with radial gap ranges from 5-20 mm were acquired and plotted in Figure 9. Table 5 lists the best efficiency points' (BEP) efficiency, head, and shaft power of different schemes. 


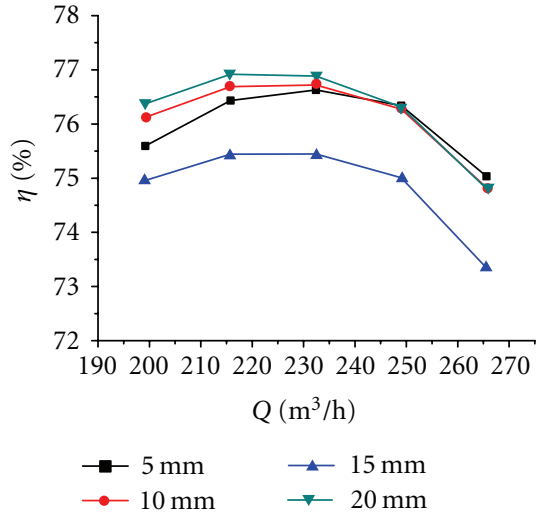

(a)

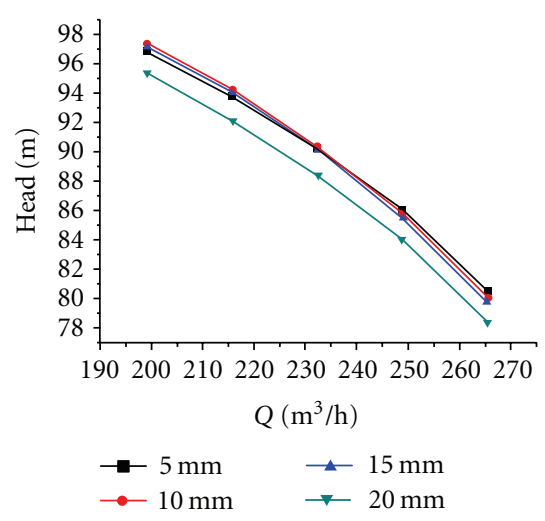

(b)

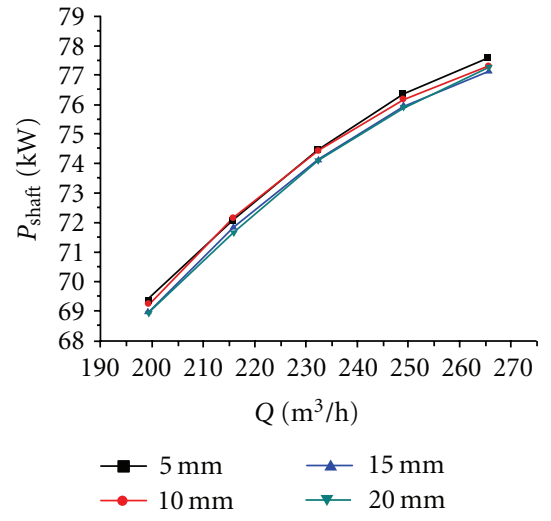

(c)

Figure 9: (a) $Q-\eta$ curve, (b) $Q-H$ curve, and (c) $Q-P_{\text {shaft }}$ curve.

TABLE 5: List of pump BEPs with different radial gaps.

\begin{tabular}{lcccc}
\hline Radial gap & Capacity $\left(\mathrm{m}^{3} / \mathrm{h}\right)$ & Efficiency $(\%)$ & Head $(\mathrm{m})$ & Shaft power $(\mathrm{kW})$ \\
\hline $5 \mathrm{~mm}$ & 232.4 & 76.63 & 90.20 & 74.47 \\
$10 \mathrm{~mm}$ & 232.4 & 76.72 & 90.31 & 74.15 \\
$15 \mathrm{~mm}$ & 215.8 & 76.92 & 94.08 & 71.85 \\
$20 \mathrm{~mm}$ & 232.4 & 75.45 & 88.37 & 74.10 \\
\hline
\end{tabular}

As is demonstrated in Figure 9(a) and Table 5, there is an optimal radial gap for the design of pump to achieve high efficiency, and the highest efficiency is reached when the radial gap is $15 \mathrm{~mm}$. As the radial gap is increased from $5 \mathrm{~mm}$ to $15 \mathrm{~mm}$, pump BEP increases at a neglectable level. As the radial gap continues to increase, its efficiency begins to drop.

Figures 9(b) and 9(c) indicate that when the radial gap increases from $5 \mathrm{~mm}$ to $15 \mathrm{~mm}$, the variation of pump pressure head and shaft power is small. As the radial gap increases to $20 \mathrm{~mm}$, pump pressure head begins to drop.

\section{Conclusions}

Volute as an important stationary hydraulic part of pump performs the function of gathering high-velocity fluid, transferring velocity energy into pressure energy. Research on design method of volute has significant meaning to the improvement of pump's efficiency.

Volute throat area is an important geometric parameter in pump design. A large volute throat area could offset pump efficiency curve to large flow, widen pump high-efficiency range, and smooth pump head curve. Also, it is observed that there is a slight drop of the highest efficiency with the increase of throat area.

Research on different volute cross-section shapes to pump performance shows that the cross-section that generates the highest efficiency among the four usual types of cross-section shapes is round. Pumps with rectangular and trapezoid shape volute have almost the same efficiency. Round cross-section shape is the best option for the volute design to achieve high efficiency.
Five different design rules of volute spiral development areas are investigated. Research results indicate that the volute spiral development areas when designed according to the constant swirl rule has the highest efficiency. And the second highest efficiency is attained when volute is designed according to constant velocity rule, slightly lower than that of constant swirl.

Investigation into the radial gap between impeller and volute tongue illustrates that there is an optimal radial gap for a high-efficiency pump design. The optimal radial gap of this design parameter is $15 \mathrm{~mm}$, which is 0.053 -times of the impeller diameter, or that the volute base circle diameter is 1.107-times of the impeller outlet diameter.

A design method of high-efficiency pump volute is developed through numerical investigations into volute geometric parameters to pump performance. This research provides guidance to high-efficiency pump volute design.

\section{Nomenclature}

$\begin{array}{ll}A: & \text { Volute throat area, }\left(\mathrm{mm}^{2}\right) \\ b_{2}: & \text { Impeller outlet width, }(\mathrm{mm}) \\ D_{1}: & \text { Pump inlet diameter, }(\mathrm{mm}) \\ D_{2}: & \text { Impeller outlet diameter, }(\mathrm{mm}) \\ D_{3}: & \text { Volute base circle diameter, }(\mathrm{mm}) \\ D_{4}: & \text { Volute outlet diameter, }(\mathrm{mm}) \\ H: & \text { Head, }(\mathrm{m}) \\ n: & \text { Rotational speed, }(\mathrm{r} / \mathrm{min}) \\ P_{\text {shaft }}: & \text { Shaft power, }(\mathrm{kW}) \\ \mathrm{Q}: & \text { Volume flow rate, }\left(\mathrm{m}^{3} / \mathrm{h}\right) \\ z: & \text { Blade number }\end{array}$




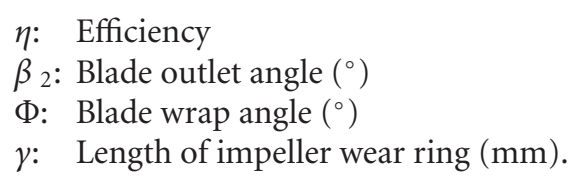

\section{Acknowledgments}

Special thanks are given to the national projects titled "The Exploitation and Utilization of Hydraulic Power Recovery Turbines (2009GJC10007)"and Doctoral innovation foundation of Jiangsu University entitled "Research on HighPressure Fluid Power Recovery Device of Pump as Turbine (CX10B_012X).”

\section{References}

[1] X.-B. Zhao and H.-Y. Xu, "Research and development on volute of hydraulic machinery," Transactions of the Chinese Society for Agricultural Machinery, vol. 34, no. 2, pp. 136-140, 2003.

[2] W.-J. Deng and W.-L. Chu, "Research on the interaction of impeller and volute to the performance of pump," China Petroleum Machinery, vol. 36, no. 3, pp. 9-12, 2008.

[3] Y.-L. Chen and H.-J. Wei, "Influence of variable area rule on the hydraulic performance of the full spiral casing," Large Electric Machine and Hydraulic Turbine, vol. 3, pp. 36-39, 2007.

[4] K. Majidi, "Numerical study of unsteady flow in a centrifugal pump," Journal of Turbomachinery, vol. 127, no. 2, pp. 363371, 2005.

[5] S. Derakhshan and A. Nourbakhsh, "Theoretical, numerical and experimental investigation of centrifugal pumps in reverse operation," Experimental Thermal and Fluid Science, vol. 32, no. 8, pp. 1620-1627, 2008.

[6] J. Denton and W. Dawes, "CFD for turbomachinery design," in Presented at ImechE Semiar "The Successful Exploration of CFD in Turbomachinery Design", London, UK, March 1998.

[7] S. M. Miner, "Evaluation of blade passage analysis using coarse grids," Journal of Fluids Engineering, vol. 122, no. 2, pp. 345348, 2000.

[8] R. Spence and J. Amaral-Teixeira, "Investigation into pressure pulsations in a centrifugal pump using numerical methods supported by industrial tests," Computers \& Fluids, vol. 37, no. 6, pp. 690-704, 2008.

[9] X.-F. Guan, Pump Handbook, Yuhang Press, 1995.

[10] D.-K. Liu, Hydraulic Turbine, China Water Power Press, 1996.

[11] J. Kurokawa, K. Matsumoto et al., "Development of high efficiency volute pump of very low specific speed," in Proceedings of the 6th Asian International Conference on Fluid Machinery, pp. 250-255, Beijing, China, 2000. 

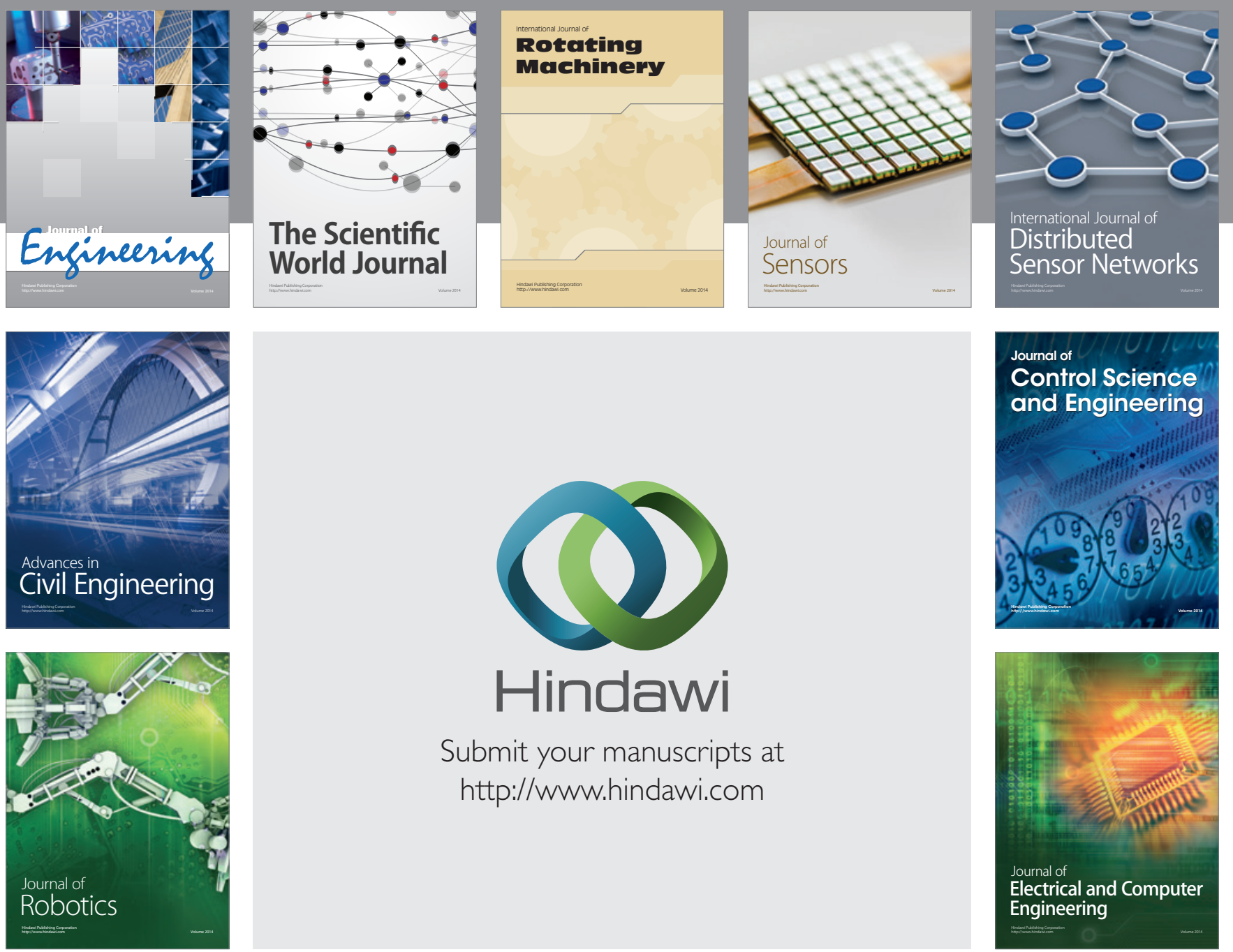

Submit your manuscripts at

http://www.hindawi.com
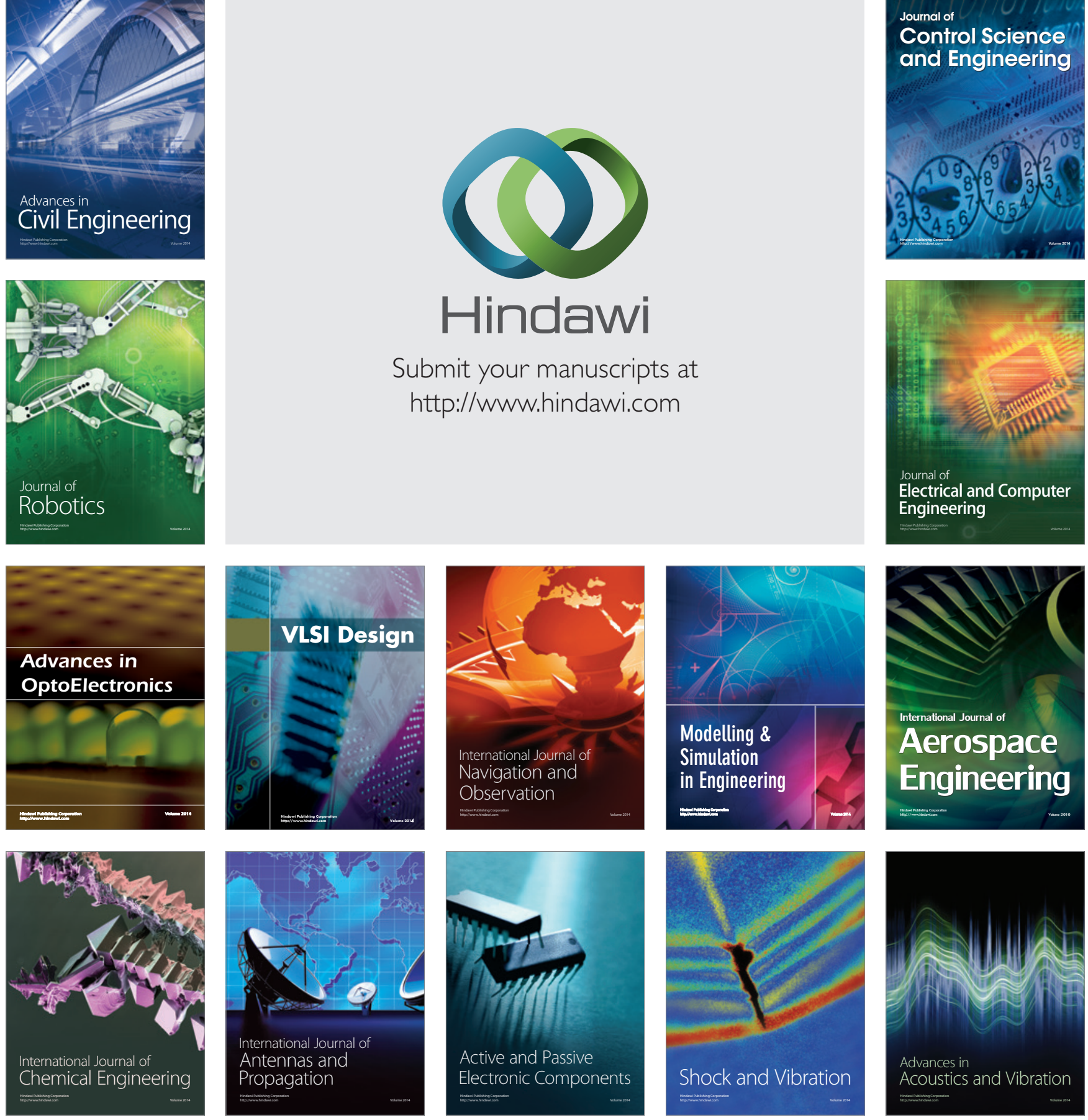